\title{
Low-Pressure Small MEMS Accelerometer Using Sigma-Delta Modulation ${ }^{\dagger}$
}

\author{
Vasco Lima ${ }^{1, *}$, Jorge Cabral ${ }^{2}$, Burkhard Kuhlmann ${ }^{3}$ and Luis Alexandre Rocha ${ }^{1,4}$ \\ 1 CMEMS-UM, University of Minho, 4800-058 Guimarães, Portugal; 1rocha@dei.uminho.pt \\ 2 ALGORITMI CENTER, University of Minho, 4800-058 Guimarães, Portugal; jcabral@dei.uminho.pt \\ 3 Automotive Electronics, Robert Bosch GmbH, 72762 Reutlingen, Germany; \\ Burkhard.Kuhlmann@de.bosch.com \\ 4 INL, International Iberian Nanotechnology Laboratory, 4715-330 Braga, Portugal \\ * Correspondence: id5998@alunos.uminho.pt; Tel.: +351-253-510-190 \\ + Presented at the Eurosensors 2018 Conference, Graz, Austria, 9-12 September 2018.
}

Published: 13 November 2018

\begin{abstract}
Encapsulation of MEMS accelerometers in vacuum is advantageous, since it allows integration with other sensors, leading to size and cost reduction. One technique to operate MEMS accelerometers in vacuum is the use of closed-loop Sigma-Delta modulation, which has many advantages. In this paper, the design of a vacuum encapsulated small size MEMS accelerometer $(0.2$ $\mathrm{mm}^{2}$ ) and the preliminary measured results are presented. Experimental devices were fabricated and operated in 2nd and 3rd order Sigma-Delta modulators, achieving a noise figure of $389 \mu \mathrm{g} / \mathrm{VHz}$ for a bandwidth of $200 \mathrm{~Hz}$ and a measurement range of at least $\pm 1 \mathrm{~g}$.
\end{abstract}

Keywords: MEMS; accelerometer; Sigma-Delta; low-pressure

\section{Introduction}

Vacuum encapsulation of MEMS accelerometers can be advantageous, since it enables integration with other MEMS sensors that require vacuum operation, such as gyroscopes, resonators, barometers, and magnetometers. This kind of integration allows for size and cost reduction, improvement of the mechanical Brownian noise performance, and realization of new devices, such as ten degrees-of-freedom (DOF) sensing microsystems, to accurately map the position of objects in space, and six DOF inertial measurement units, for image stabilization in digital cameras, or electronic stability control systems in robotics and automobiles [1]. However, low damping makes the accelerometer more difficult to operate.

Typically, MEMS accelerometers encapsulated in vacuum are operated in a closed-loop or use frequency modulation. Frequency modulated accelerometers require large polarization voltages and complex compensation techniques [1]. In other hand, closed-loop operated accelerometers don't have these problems and have some additional advantages, such as improved bandwidth, linearity, and dynamic range [2,3]. Sigma-Delta modulation (first used by H. Inose et al. in 1962 [4]), is well suited for low-frequency signals, such as environmental accelerations, and its output signal is inherently digital, leading to the realization of high-performance accelerometers [5-7].

In the literature, one can find vacuum encapsulated high performance MEMS accelerometers using Sigma-Delta modulation, however, all present a common characteristic: large size proof-mass [1,8-11]. An accelerometer with a large seismic mass, in addition to reducing the size advantages of integration with other sensors, may present some additional problems, such as reduced full-scale range and added complexity in the manufacturing process, leading to cost increase [12]. 
In this work, a small size and low-pressure MEMS accelerometer, which is operated with a closed-loop Sigma-Delta modulator implemented in an FPGA, is presented. Preliminary experimental data is shown.

\section{System Design}

Part of the read-out circuit (digital lock-in amplifier and low-pass IIR filter), as well as the phase lag compensator and Sigma-Delta modulator were implemented in FPGA, as seen in the diagram presented in Figure 1. This approach enables to perform a fast and effective fine tuning of the phase lag compensator and the feedback gain for the third order modulator (kf), which is typically performed by simulation [6]. This aims to achieve stability of the loop, more difficult for low-damping devices, and to improve the system's performance.

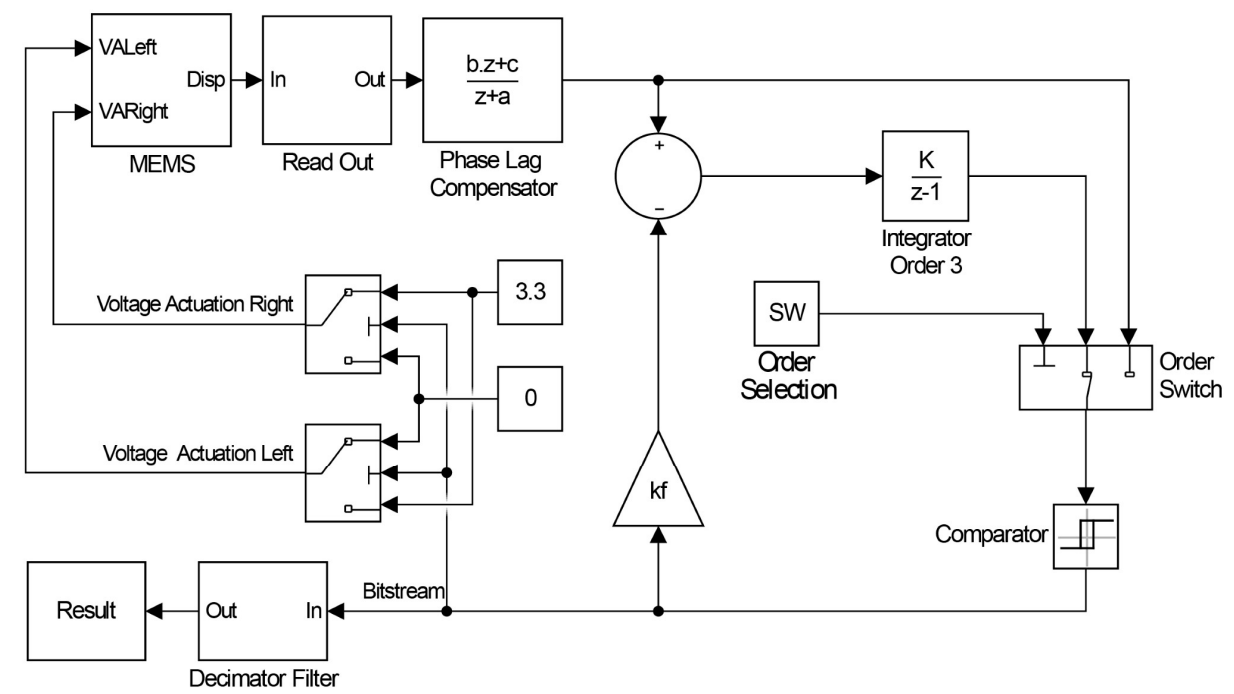

Figure 1. Implemented configurable Sigma-Delta architecture.

Six units of the MEMS accelerometer, shown in Figure 2, were fabricated and encapsulated with a pressure of $1.4 \mathrm{mBar}$ using a Bosch MEMS process. Their size is approximately $450 \times 450 \mu \mathrm{m}$, with a suspended mass of $2.83 \mu \mathrm{g}$, an elastic coefficient on the sensing axis of $0.75 \mathrm{~N} / \mathrm{m}$, and a nominal sensing capacity of $246 \mathrm{fF}$ (for a gap at rest of $1.6 \mu \mathrm{m}$ ). Table 1 presents the theoretical and measured experimental values, as well as the respective error, for the pull-in voltage, resonant frequency, and quality factor of each fabricated device.

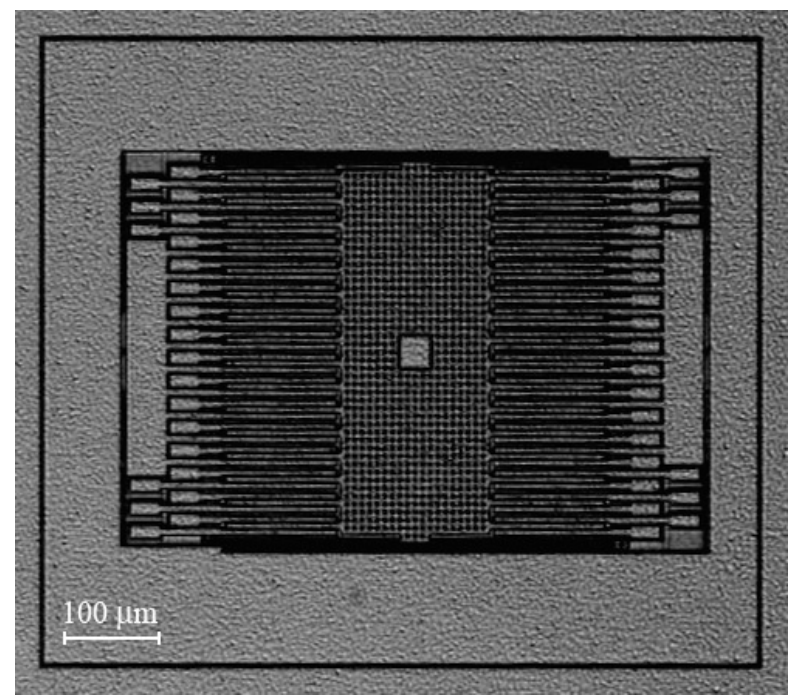

Figure 2. Fabricated MEMS accelerometer. 
Table 1. Mechanical characterization of the fabricated devices.

\begin{tabular}{ccccccc}
\hline & $\begin{array}{c}\text { Pull-in } \\
\text { Voltage } \\
(\mathbf{V})\end{array}$ & $\begin{array}{c}\text { Pull-in } \\
\text { Voltage } \\
\text { Error (\%) }\end{array}$ & $\begin{array}{c}\text { Resonant } \\
\text { Frequency } \\
\mathbf{( H z )}\end{array}$ & $\begin{array}{c}\text { Resonant } \\
\text { Frequency } \\
\text { Error (\%) }\end{array}$ & Quality Factor & $\begin{array}{c}\text { Quality } \\
\text { Factor } \\
\text { Error (\%) }\end{array}$ \\
\hline Theorectical & 1.962 & - & 2591 & - & 58.6 & - \\
Device 1 & 1.910 & 2.7 & 2498 & 3.6 & 61.1 & 4.3 \\
Device 2 & 1.839 & 3.5 & 2535 & 2.2 & 58.3 & 0.5 \\
Device 3 & 1.892 & 3.6 & 2537 & 2.1 & 56.0 & 4.4 \\
Device 4 & 1.893 & 3.5 & 2543 & 1.9 & 53.9 & 8.0 \\
Device 5 & 1.888 & 3.8 & 2526 & 2.5 & 61.4 & 4.8 \\
Device 6 & 1.888 & 3.8 & 2536 & 2.1 & 58.6 & 0.0 \\
\hline
\end{tabular}

The closed-loop operates with a sampling frequency of $454 \mathrm{kHz}$, the one-bit DAC uses a $3.3 \mathrm{~V}$ actuation voltage, and the decimation filter outputs a 20-bit result with a decimation ratio of 2272 . The phase lag compensator, which was implemented based in the approach followed by William Messner in [13], was tuned for $64^{\circ}$ phase advance at $9105 \mathrm{~Hz}$, to minimize the delay introduced by the MEMS device and low-pass filter. Figure 3 depicts the experimental setup used to achieve the presented results.

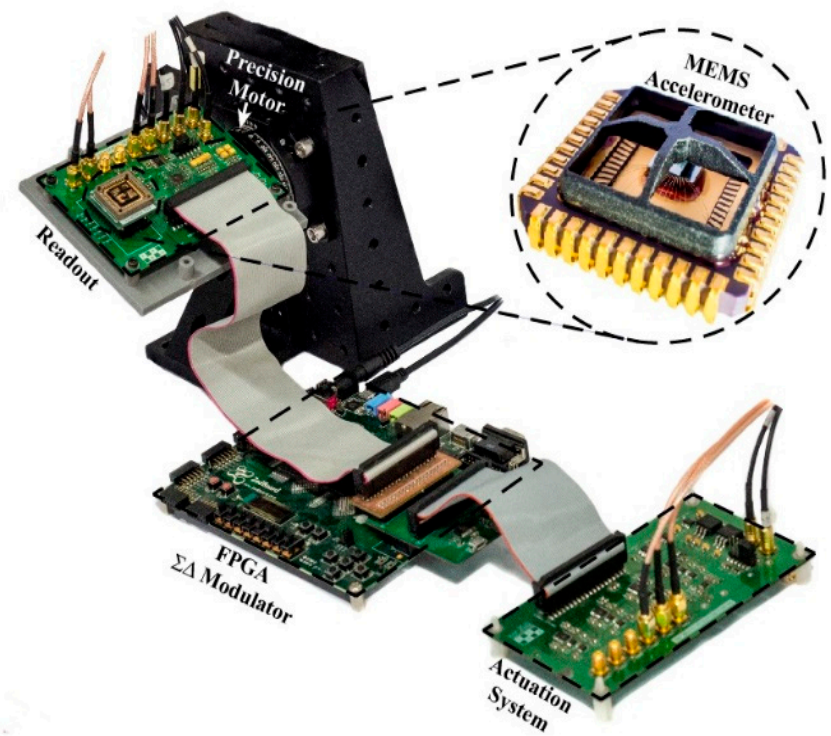

Figure 3. Experimental setup.

\section{Results}

The performance of the system was evaluated using 2nd and 3rd order Sigma-Delta modulators. For second order, the feedback gain is irrelevant, since the MEMS accelerometer behaves as the second order integrator, however, for the third modulation order, the kf used was 382.

Figures 4 and 5 present the Allan variance and the power spectrum density of the output bitstream, respectively, obtained for all six devices. The experimental sensitivities, measured for an acceleration range of $\pm 1 \mathrm{~g}$, are presented in Table 2 . As expected, the overall results highlight the better performance obtained by the higher-order modulation. 


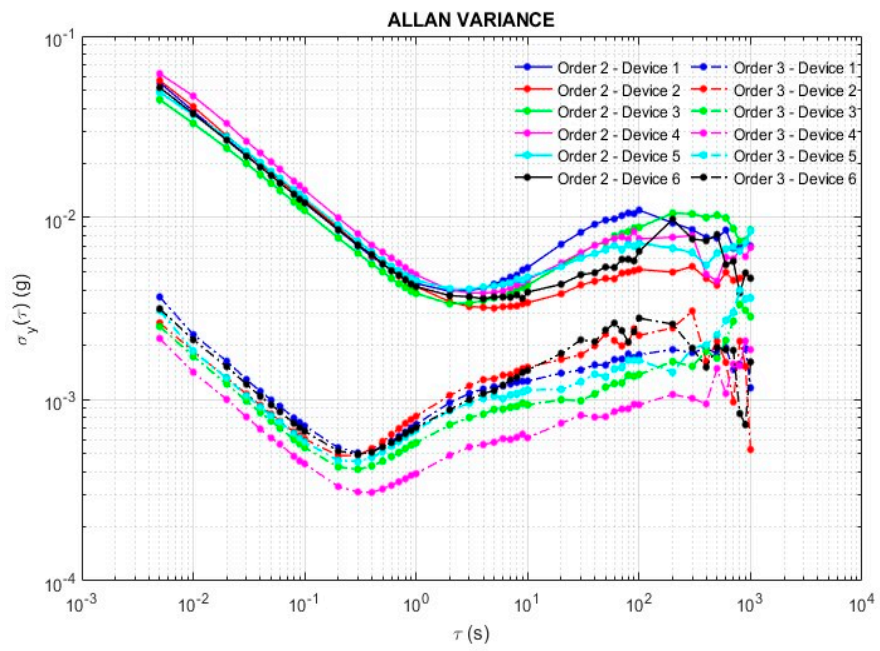

Figure 4. Allan variance.
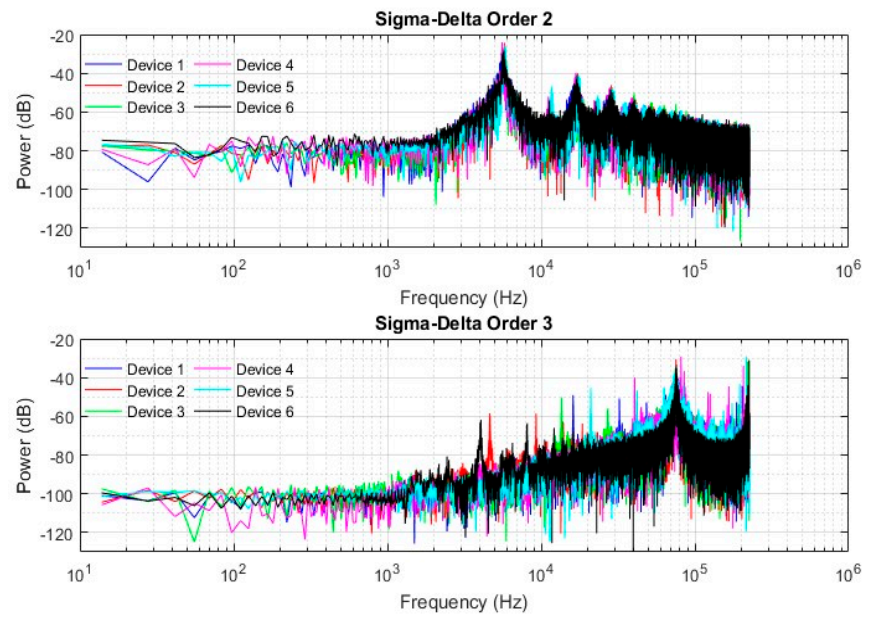

Figure 5. Power spectrum density.

Table 2. Experimental sensitivity.

\begin{tabular}{ccc}
\hline & \multicolumn{2}{c}{ Sensitivity (bit/g) } \\
\hline & Order 2 & Order 3 \\
\hline Device 1 & 14.511 & 13.640 \\
Device 2 & 14.437 & 13.543 \\
Device 3 & 14.463 & 13.690 \\
Device 4 & 14.330 & 13.760 \\
Device 5 & 14.353 & 13.651 \\
Device 6 & 14.496 & 13.502 \\
\hline
\end{tabular}

As seen in Figure 4, the best noise level achieved with a 2 nd order modulator was $3.82 \mathrm{mg} / \mathrm{VHz}$ for device 3, and with a 3rd order modulator, device 4 achieved a noise level of $389 \mu \mathrm{g} / \sqrt{ } \mathrm{Hz}$. In Figure 5 , is visible that the noise is being shaped into the higher frequencies for the higher-order modulation. These results are in accordance with the fundamental theory of Sigma-Delta modulators [14].

\section{Conclusions and Future Work}

A small size and low-pressure MEMS accelerometer using Sigma-Delta modulation, was presented in this paper. The closed-loop was implemented in FPGA, enabling the fast and accurate tuning of the parameters in an experimental environment. The best noise figure achieved was 389 $\mu \mathrm{g} / \sqrt{\mathrm{Hz}}$ for a bandwidth of $200 \mathrm{~Hz}$ and a minimum measurement range of $\pm 1 \mathrm{~g}$. As expected, the 
overall results highlight the better performance achieved by the higher-order modulator. The platform is now going to be used for testing different modulation orders and architectures aiming better performance.

Acknowledgments: The first author is supported by FCT- Fundação para a Ciência e Tecnologia through the grant PDE/BDE/114563/2016. This work is supported by FCT with the reference project UID/EEA/04436/2013, COMPETE 2020 with the code POCI-01-0145-FEDER-006941.

\section{References}

1. Chen, F.; Yuan, W.; Chang, H.; Zeimpekis, I.; Kraft, M. Low noise vacuum MEMS closed-loop accelerometer using sixth-order multi-feedback loops and local resonator sigma delta modulator. In Proceedings of the IEEE 27th International Conference on Micro Electro Mechanical Systems (MEMS), San Francisco, CA, USA, 26-30 January 2014; pp. 761-764.

2. Amini, B.V.; Abdolvand, R.; Ayazi, F. A 4.5-mW Closed-Loop $\Delta \sum$ Micro-Gravity CMOS SOI Accelerometer. IEEE J. Solid-State Circuits 2006, 41, 2983-2991.

3. Xu, H.; Liu, X.; Yin, L. A Closed-Loop $\Sigma \Delta$ Interface for a High-Q Micromechanical Capacitive Accelerometer with $200 \mathrm{ng} / \mathrm{JHz}$ Input Noise Density. IEEE J. Solid-State Circuits 2015, 50, 2101-2112.

4. Inose, H.; Yasuda, Y.; Murakami, J. A Telemetering System by Code Modulation $-\Delta-\Sigma$ Modulation. IRE Trans. Space Electron. Telem. 1962, SET-8, 204-209.

5. Zwahlen, P.; Dong, Y.; Nguyen, A.M.; Rudolf, F.; Stauffer, J.M. Breakthrough in high performance inertial navigation grade Sigma-Delta MEMS accelerometer. In Proceedings of the IEEE/ION Position, Location and Navigation Symposium, Myrtle Beach, SC, USA, 23-26 April 2012; Volume 37, pp. 15-19.

6. Wilcock, R.; Kraft, M. Genetic Algorithm for the Design of Electro-Mechanical Sigma Delta Modulator MEMS Sensors. Sensors 2011, 11, 9217-9232.

7. Zhao, C.; Kazmierski, T.J. An automated design flow for MEMS accelerometers with Sigma-Delta control. In Proceedings of the IEEE International Conference on Information and Automation, Harbin, China, 20-23 June 2010; pp. 559-564.

8. Dong, Y.; Zwahlen, P.; Nguyen, A.M.; Frosio, R.; Rudolf, F. Ultra-high precision MEMS accelerometer. In Proceedings of the 16th International Solid-State Sensors, Actuators and Microsystems Conference, Beijing, China, 5-9 June 2011; pp. 695-698.

9. Lemkin, M.; Boser, B.E. A three-axis micromachined accelerometer with a CMOS position-sense interface and digital offset-trim electronics. IEEE J. Solid-State Circuits 1999, 34, 456-468.

10. Nakamura, S. MEMS Inertial Sensor Toward Higher Accuracy \& Multi-Axis Sensing. In Proceedings of the IEEE Sensors, 31 October-3 November 2005; pp. 939-942.

11. Henrion, W.; DiSanza, L.; Ip, M.; Terry, S.; Jerman, H. Wide dynamic range direct digital accelerometer. In Proceedings of the IEEE 4th Technical Digest on Solid-State Sensor and Actuator Workshop, Hilton Head Island, SC, USA, 4-7 June 1990; pp. 153-157.

12. Sonmez, U.; Kulah, H.; Akin, T. A fourth order unconstrained $\Delta \sum$ capacitive accelerometer. In Proceedings of the 16th International Solid-State Sensors, Actuators and Microsystems Conference, Beijing, China, 5-9 June 2011; pp. 707-710.

13. Messner, W. Formulas for asymmetric lead and lag compensators. In Proceedings of the 2009 American Control Conference, St. Louis, Missouri, USA, 10-12 June 2009; pp. 3769-3774.

14. Dong, Y.; Kraft, M.; Redman-White, W. Higher order noise-shaping filters for high-performance micromachined accelerometers. IEEE Trans. Instrum. Meas. 2007, 56, 1666-1674.

(C) 2018 by the authors; Licensee MDPI, Basel, Switzerland. This article is an open access article distributed under the terms and conditions of the Creative Commons Attribution (CC BY) license (http://creativecommons.org/licenses/by/4.0/). 